\title{
Clustering Based Pruning for Statistical Criticality Computation under Process Variations
}

\author{
Hushrav D Mogal*, Haifeng Qian ${ }^{\dagger}$, Sachin S Sapatnekar* and Kia Bazargan* \\ *Department of Electrical and Computer Engineering, University of Minnesota, \\ Minneapolis, MN 55455, \{mhush, sachin, kia\}@umn.edu \\ †IBM Research, Yorktown Heights, NY, qianhaifeng@us.ibm.com
}

\begin{abstract}
We present a new linear time technique to compute criticality information in a timing graph by dividing it into "zones". Errors in using tightness probabilities for criticality computation are dealt with using a new clustering based pruning algorithm which greatly reduces the size of circuitlevel cutsets. Our clustering algorithm gives a $150 \mathrm{X}$ speedup compared to a pairwise pruning strategy in addition to ordering edges in a cutset to reduce errors due to Clark's $M A X$ formulation. The clustering based pruning strategy coupled with a localized sampling technique reduces errors to within $5 \%$ of Monte Carlo simulations with large speedups in runtime.
\end{abstract}

\section{Introduction AND PREVIOUS WORK}

With scaling of technology, process parameter variations render the circuit delay as unpredictable [6], making sign-off ineffective in assuring against chip failure. Recent works concerning Statistical Static Timing Analysis (SSTA) in [1], [9] deal with this issue by treating the delay of gates and interconnects as random variables with Gaussian distributions, and predict the mean and variance of circuit delay to within a few percents.

With process variations in effect, no one path dominates the delay of the circuit [5]. Work in [9] proposes the concept of edge(node) criticality, which is the probability that an edge(node) lies on the critical path in a manufactured chip. Works like [2], [5], [10] compute the criticality probability of edges in a timing graph, using a canonical first order delay model.

In [9], the authors perform a reverse traversal of the timing graph assuming independence of edge criticalities despite structural and spatial correlations in the circuit. Subsequently, the work in [5] used sensitivity matrices to compute the criticalities of nodes and edges in the timing graph, with complexity potentially cubic in the number of principal components of the circuit due to the matrix multiplications involved. Work in [2] perturbs gate delays to compute its effect on the circuit output delay, using cutsets to simplify computation. The quadratic time complexity of this approach is reduced to linear in [10], where the criticality of edges in a cutset is computed using a balanced binary partition tree.

Our contributions are twofold. We propose a linear time algorithm to compute the criticality probability of edges (nodes) in a timing graph using the notion of cutsets. Edges crossing multiple cutsets are dealt with using a novel zone based approach, similar to [11], where old computations are reused to the greatest possible extent. We develop a clustering based pruning algorithm to effectively eliminate a large number of non-competing edges in cutsets of several thousand edges. The proposed clustering scheme helps order the $M A X$ operations in a set, a source of significant error as shown in [8]. Localized sampling on the pruned cutset further reduces errors in edge criticalities to within $5 \%$ of Monte Carlo simulations, with large speedups in run-time when compared to a pairwise pruning strategy.

\section{BACKGROUND}

\section{A. Correlation Model and SSTA}

We use the spatial correlation model in [1] to model intra-die parameter correlations. Briefly, the chip is divided into a uniform grid in which gates in a grid square have perfect correlation and gates in far-away grid squares have weak correlations. A covariance matrix of size equal to the number of grid squares is obtained for each modeled parameter. We model the length and width of gates, and the thickness, width and inter-layer dielectric thickness for interconnects. Process parameters are assumed Gaussian as in [1], [9] and using the principal component analysis (PCA) technique each correlated parameter is expressed as a sum of independent normal random variables, or principal components (PCs). Delays of edges in the timing graph are expressed in terms of the PCs and SSTA is then performed by a forward propagation on the timing graph. For more details, readers are referred to [1].

\section{B. Definitions}

Definition II.1 (Timing Graph). A timing graph $G(V, E)$ of a circuit is a directed acyclic graph with $V$ nodes representing gate terminals and $E$ edges representing connections between them. Primary inputs and outputs are connected, respectively, to a virtual source node, $v_{s}$, and a virtual sink node, $v_{t}$. The delay of an edge in $G$ is represented by a probability density function $(p d f)$

This work was supported in part by the SRC under award 2007-TJ-1572.

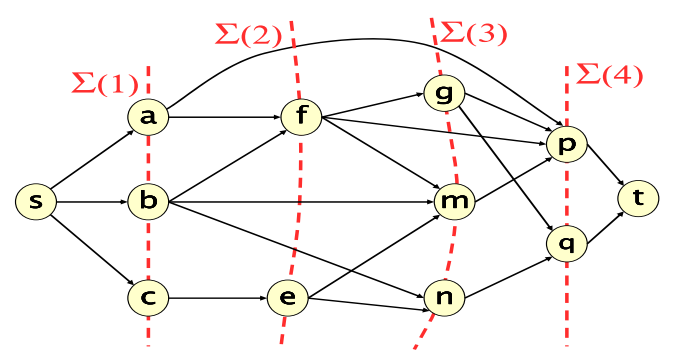

Fig. 1. Example timing graph $G$ (depth $L$ ) illustrating cutsets $\Sigma(1)-\Sigma(4)$.

Definition II.2 (Cutset). A cutset $\Sigma$ is a set of edges/nodes in $G$ such that every $v_{s}$ to $v_{t}$ path passes through one and only one member of $\Sigma$.

Definition II.3 (Arrival Time (Required Time)). The arrival time $A T$ (required time $R T$ ) at an edge/node in $G$ is the maximum delay from any primary input (output) to the edge/node. Like delays, these are also $p d f s$.

Definition II.4 (Path Delay). The path delay of an edge/node (hereon, also referred to as edge delay) $i$ in $G$ is defined as $e_{i}=A T_{i}+R T_{i}$ and is a $p d f$. Each path delay $e_{i}$ is represented in a canonical form in terms of independent principal components (PCs) $p_{j}$, as

$$
e_{i}=\mu_{i}+\sum_{j=1}^{j=k} a_{i j} \cdot p_{j}
$$

Here $a_{i j}$ is the coefficient of PC $j$ for edge $i$ and $k$ is the total number of PCs. The mean of the edge delay is given by $\mu_{i}$.

Definition II.5 (Complementary Path Delay). Given a cutset, $\Sigma$, in a timing graph $G$, the complementary path delay of an edge/node $k \in \Sigma \subset G, e_{k}{ }^{\prime}$, is defined as the $p d f$ of $M A X\left(\forall e_{i}: e_{i} \in \Sigma, e_{i} \neq e_{k}\right)$.

Definition II.6 (Local Criticality). The local criticality (also referred to as tightness probability [9]) $\tau_{i j}$ of edge $e_{i}$ with respect to $e_{j}$, with means $\mu_{i}, \mu_{j}$ and standard deviations $\sigma_{i}, \sigma_{j}$ respectively, and correlation $\rho_{i j}$, is given by

$$
\tau_{i j}=\Phi\left(\frac{\mu_{i}-\mu_{j}}{\theta}\right)
$$

where $\theta=\sqrt{\sigma_{i}^{2}+\sigma_{j}^{2}-2 \cdot \rho_{i j} \cdot \sigma_{i} \cdot \sigma_{j}}$. The degree of domination of edge $i$ over $j$ is represented by $\tau_{i j} . \Phi$ is the distribution ( $\left.c d f\right)$ of a unit normal random variable $\mathcal{N}(0,1)$. It can be shown that $\tau_{j i}=1-\tau_{i j}$.

Definition II.7 (Global Criticality). The global criticality $T_{i}$ (also referred to as criticality hereon) of edge $e_{i}$ in cutset $\Sigma$ is the probability that it has maximum delay among all the edges in the cutset, i.e.,

$$
=\operatorname{Pr}\left(e_{i} \geq e_{i}{ }^{\prime}\right) \quad \text { (see Def. II.5) }
$$

$T_{i}$ is also referred to as the criticality probability of $e_{i}$. It follows that the global criticality of an edge $e_{i}$ in $\Sigma$ cannot be greater than its local criticality with respect to any other edge in $\Sigma$, i.e.,

$$
T_{i} \leq \tau_{i j} \quad\left\{\forall e_{j} \in \Sigma, \quad e_{j} \neq e_{i}\right\}
$$

Definition II.8 $\left(\mathbf{M A X}_{\theta}\right)$. The maximum $M A X_{\theta}$ of two normal random variables $e_{i}$ and $e_{j}$ in canonical form using Clark's formulation [3] is given by $c=\operatorname{MAX}_{\theta}(i, j)$, where $\phi$ is the density $(p d f)$ function of $\mathcal{N}(0,1)$ and

$$
\begin{aligned}
\mu_{c}= & \tau_{i j} \cdot \mu_{i}+\tau_{j i} \cdot \mu_{j}+\theta \cdot \phi\left(\frac{\mu_{i}-\mu_{j}}{\theta}\right) \\
\sigma_{c}^{2}= & \tau_{i j} \cdot\left(\sigma_{i}^{2}+\mu_{i}^{2}\right)+\tau_{j i} \cdot\left(\sigma_{j}^{2}+\mu_{j}^{2}\right) \\
& +\left(\mu_{i}+\mu_{j}\right) \cdot \theta \cdot \phi\left(\frac{\mu_{i}-\mu_{j}}{\theta}\right)-\mu_{c}^{2} \\
c_{k}= & \tau_{i j} \cdot a_{i k}+\tau_{j i} \cdot a_{j k}
\end{aligned}
$$

\section{Statistical CRiticality Computation}

This section describes a quadratic time complexity statistical criticality (SC) algorithm followed by details of our linear time approach. 


\section{A. Cutset Computation}

Fig. 1 illustrates cutset computation on a timing graph $G$. We topologically traverse $G$, ordering nodes as per their levels, $l$, denoted $\Sigma_{n}(l)$, and edges crossing $l$ denoted $\Sigma_{e}(l)$. For instance, $\Sigma_{n}(2)=\left\{n_{e}, n_{f}\right\}$ and $\Sigma_{e}(2)=$ $\left\{e_{a p}, e_{b m}, e_{b n}\right\} . \Sigma(l)=\Sigma_{n}(l) \cup \Sigma_{e}(l)$ forms a cutset by Def. II.2 since every $v_{s}$ to $v_{t}$ path in $G$ must pass through at least one member of $\Sigma(l)$ and its elements are disjoint. Our aim is to compute the criticalities of all edges in $G$, using Def. II.7. Towards this end, the topological level-enumerated cutsets are necessary and sufficient since they cover all the nodes and edges in $G$. The number of cutsets equals the number of levels $L$ in $G$. To compute the criticality of all edges in $G$, we substitute nodes in $\Sigma_{n}$ with their fanout edges. For instance, cutset $2, \Sigma(2)=\left\{e_{e m}, e_{e n}, e_{f g}, e_{f p}, e_{f m}, e_{a p}, e_{b m}, e_{b n}\right\}$.

\section{B. BSC: Basic SC Algorithm}

The simplistic approach BSC shown in Algorithm 1 computes the global criticalities of all edges in timing graph $G$. Step 1 performs a forward and reverse SSTA to compute path delays $(A T+R T)$ of all edges (nodes) in $G$, followed by a topological ordering of $G$ into levels to compute its cutsets in $\Sigma$. Steps 3-8 compute the criticality of each edge in $\Sigma$.

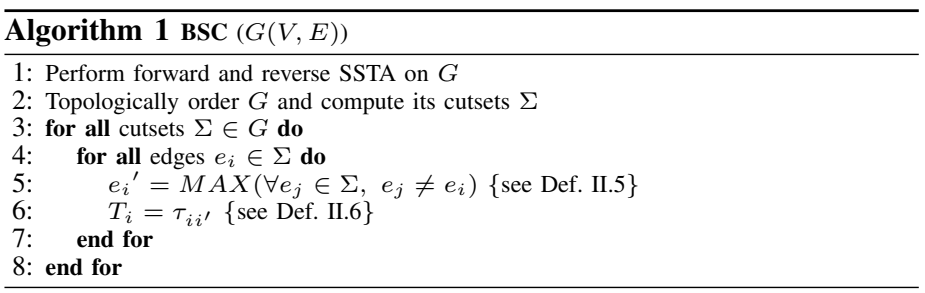

Definition III.1 (mc-edge). An mc-edge is an edge with end level at leas one greater than its start level. With our level enumerated cutsets, these are edges which cross over at least one cutset. In Fig. 1, $e_{a p}, e_{b m}$ and $e_{b n} \in \Sigma_{e}(2)$ are a set of mc-edges crossing level 2. However, edges like $e_{a f}$ and $e_{b f}$ are not mc-edges since they start at level 2 and end at level 3 with no cross over.

Due to mc-edges, each cutset $\Sigma$ can potentially contain $O(E)$ edges. Moreover, since Step 5 computes the complementary path delay of an edge in time linear in the size of $\Sigma$, over all cutsets in $G$, Algorithm 1 has a complexity of $O\left(L E^{2}\right)$. Sections III-C and III-D discuss methods to reduce the time complexity of the basic approach.

\section{Linear Time Book-Keeping}

Definition III.2 (Ordered Lists). Given an arbitrary set $\Sigma=\left\{e_{1}, e_{2}, \ldots e_{n}\right\}$ of $n$ random variables, we define ordered lists $\Upsilon_{F}$ and $\Upsilon_{R}$ as follows

$$
\begin{aligned}
& \Upsilon_{F}(i)=\operatorname{MAX}\left(e_{1}, \ldots e_{i}\right) \\
& \Upsilon_{R}(i)=\operatorname{MAX}\left(e_{i}, \ldots e_{n}\right)
\end{aligned}
$$

The global criticality of an edge $e_{i} \in \Sigma$ (Def. II.7) is

$$
\begin{aligned}
T_{i} & =\operatorname{Pr}\left(e_{i}>\operatorname{MAX}\left(e_{1}, \ldots e_{i-1}, e_{i+1}, \ldots e_{n}\right)\right) \\
& =\operatorname{Pr}\left(e_{i}>\operatorname{MAX}\left(\Upsilon_{F}(i-1), \Upsilon_{R}(i+1)\right)\right.
\end{aligned}
$$

Computing $\Upsilon_{F}$ and $\Upsilon_{R}$ takes $2 n M A X$ operations. Eq. 8 takes two $M A X$ operations for a total of $4 n M A X$ operations over all edges in $\Sigma$. Our ordered lists can thus compute criticalities of cutset edges in linear time.

\section{Zone Computation}

Using $\Upsilon_{F}$ and $\Upsilon_{R}$, Steps 5-6 of Algorithm 1 take time $O(E)$ as compared to $O\left(E^{2}\right)$. However, since Algorithm 1 recomputes criticalities of mc-edges (Def. III.1) in every cutset $\Sigma$, over $L$ cutsets we could take $O(L E)$ time, still a considerable slowdown. Moreover, the complementary path delay (Def. II.5) for each edge $e_{i} \in \Sigma$ (excluding mc-edges) at level $l$ has in common all the mc-edges crossing $l$. The only information therefore needed, is the collective $M A X$ of these mc-edges. Zones help us compute this $M A X$.

Definition III.3 (Zone). A zone $Z_{i}$ is a maximal set of mc-edges with the end-level of any edge higher than the start-level of all edges in $Z_{i}$, i.e., edges enter a zone before any edge exits it.

We divide $G$ into zones $Z_{i}$, which contain mutually exclusive sets of mcedges and compute the $M A X$ of these edges at any level by performing a $M A X$ over all the zones. To do this however, we also need to keep track of the mc-edges which contribute to the $M A X$ of a zone (called $Z_{i M A X}$ ) at each level of $G$. This is performed using pointers $z_{i f}$ and $z_{i r}$ which maintain the history of mc-edges entering (recorded in $Z_{i F}$ ) and leaving (recorded in $\left.Z_{i R}\right)$ each zone $Z_{i}$ respectively. The authors in [10] use an array based structure to keep track of mc-edges, with no notion of a maximal set.

\section{E. ZSC: Zone Based SC Algorithm}

Our zone based criticality computation technique, ZSC, is shown in Algorithm 2. Step 2 computes zones in $G$ in time linear in the size of $\Sigma_{e}$ We then forward traverse $G$ from $v_{s}$ to $v_{t}$. Steps 4-5 update the forward and reverse history pointers of each zone $Z_{i}$, to compute $Z_{i M A X}$ in constant time. In Step 6 we compute the $M A X$ over all the currently active zones. Since we have on the order of $O(L)$ number of zones, over all levels of $G$ this step takes $O\left(L^{2}\right)$ time. Finally using the book-keeping ordered lists from Section III-C, we compute global criticalities of edges in $\Sigma$ in linear time. The overall runtime of our ZSC algorithm is therefore $O\left(E+L^{2}\right)$, which for a reasonably sized circuit is $O(E)$.

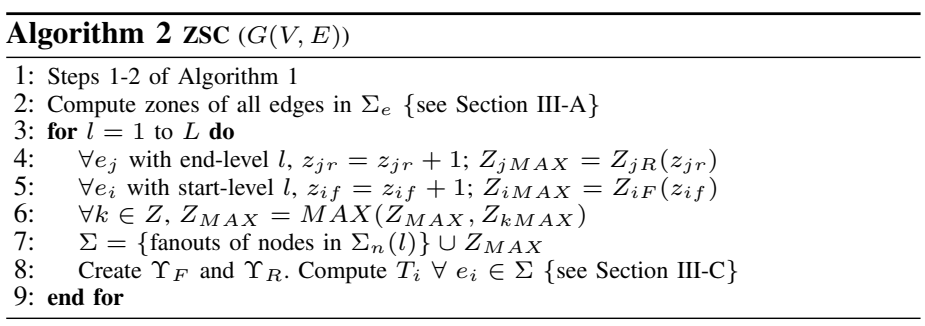

\section{ERRORS IN ZSC AND THE “ABC” PROBLEM}

Algorithm 2 was run on the ISCAS89 benchmarks to compute the global criticalities (see Def. II.7) of all edges in the timing graph $G$. We compared our implementation with a Monte Carlo (MC) simulation and noted the absolute maximum difference in criticalities of edges (denoted $\delta$ hereon). The $\delta$ was larger than $50 \%$ (for example, an edge reported by MC as $80 \%$ critical was reported by Algorithm 2 as $30 \%$ critical). As an illustration of these errors, consider a cutset $\Sigma$ with random variables $a, b$ and $c$, each with independent PCs $p_{1}$ and $p_{2}$ (where $p_{i}$ is an $\mathcal{N}(0,1)$ Gaussian), shown below,

$$
\begin{aligned}
& a=4.000+0.5000 \cdot p_{1}+0.5000 \cdot p_{2} \\
& b=3.999+0.4999 \cdot p_{1}+0.5001 \cdot p_{2} \\
& c=3.800+0.6001 \cdot p_{1}+0.3999 \cdot p_{2}
\end{aligned}
$$

Observe that $a$ and $b$ are nearly identical highly correlated random variables, and for any sample value of the $p_{i}{ }^{\prime} s, a \geq b$ (high correlation coupled with the difference in means ensures $\operatorname{Pr}(b>a) \approx 0.0)$. We ran a MC simulation with 100000 samples to determine the global criticalities of $a, b$ and $c$. Table I shows a comparison with Clark's formulation $M A X_{\theta}$ (see Def II.8). We obtained errors of $57 \%$ and $30 \%$ in the global criticality of $a$ and $b$ respectively, as shown in the last row of Table I. For better illustration purposes, Fig 2 depicts the scenario of Eq. 9, but using just one principal component (PC), $p$. We make the following observations.

TABLE I

COMPARISON OF MC AND $M A X_{\theta}$ FOR THE ABC PRoblem

\begin{tabular}{|l|l|l|l|}
\hline Method & $T_{a}$ & $T_{b}$ & $T_{c}$ \\
\hline MC & 0.923 & 0.000 & 0.077 \\
\hline Clark & 0.356 & 0.297 & 0.079 \\
\hline Error $\delta$ & $56.7 \%$ & $29.7 \%$ & $0.2 \%$ \\
\hline
\end{tabular}

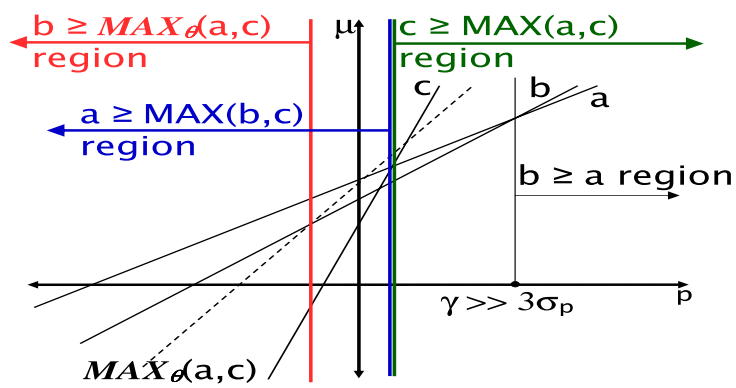

Fig. 2. A pictorial depiction of the abc example scenario with random variables $a, b$ and $c$ with one PC, $p$. The figure is not to scale.

(1) The local criticality of $b$ with respect to $a$, i.e., $\tau_{b a} \approx 0$. This is indicated by a large value of $\gamma \gg 3 \sigma_{p}$ (the region where $b \geq a$ ). Moreover, Clark's tightness probability formulation from Eq. 2 also gives $\tau_{b a} \approx 0$.

(2) Global criticality of $b, T_{b} \approx 0$. This is evident in Fig. 2 where regions $a \geq M A X(b, c)$ and $c \geq M A X(a, b)$ cover the entire probability space. 
Observations 1 and 2 are consistent with Eq. 4. Now consider global criticality of $b$, using the cutset approach. We first compute complementary path delay $b^{\prime}=M A X(a, c)$. It follows (Def. II.7) that $T_{b}=\operatorname{Pr}(b \geq$ $M A X(a, c))$. With Clark's formulation $M A X_{\theta}$, for the $M A X$, we get $T_{b}=\operatorname{Pr}\left(b \geq \operatorname{MAX}_{\theta}(a, c)\right)=0.297$.

Intuitively, for this scenario Clark's formulation is accurate with respect to local criticality $t_{b a}$ of $b$, but it overestimates global criticality $T_{b}$, and is inconsistent with Eq. 4.

Definition IV.1 (Local Errors). With respect to Clark's formulation, edge $e_{i}$ in cutset $\Sigma$ is said to have local errors iff there exists some edge $e_{j} \in \Sigma$ for which its local criticality is smaller than its global criticality, i.e.,

$$
\left\{\exists e_{j} \in \Sigma, \quad e_{j} \neq e_{i}\right\} \quad: \quad \tau_{i j}<T_{i}
$$

In other words, Eq. 4 does not hold. The difference, $T_{i}-\tau_{i j}$ is called the local error of $e_{i}$. By definition, local errors always overestimate the criticality of an edge in $\Sigma$. In our toy example, $b$ exhibits local errors with a magnitude of 0.297. Local errors were found to propagate in the ZSC algorithm, where variables (edges) like $b$ that should never have been critical, were found to have a significant global criticality.

To further see the inconsistencies of the tightness probability approach using Clark's formulation we consider the event $a \geq M A X_{\theta}(b, c)$, with $a$ represented as $a=a_{0}+\sum a_{i} p_{i}=a_{0}+a_{p}$. Variables $b$ and $c$ are represented similarly. We have,

$$
\begin{aligned}
\text { Event }\{a & \left.\geq M A X_{\theta}(b, c)\right\} \equiv \\
\tau_{b c}(a-b)+\tau_{c b}(a-c) & \geq \alpha+s\left(\tau_{b c} b_{p}+\tau_{c b} c_{p}\right)
\end{aligned}
$$

Here $\alpha$ and $s$ are factors used to equate the first two moments (mean and standard deviation) of the non-Gaussian $M A X$ with Clark's Gaussian $M A X_{\theta}$ approximation. Values of $\alpha \approx 0$ and $s \approx 0$ could result in a true event even for cases in which $b \leq a \leq c$ or $c<a<b$, overestimating the global criticality $T_{a}$ of $a$. Values of $\alpha>0$ could result in a false event even in the case that $a \geq b$ and $a \geq c$, underestimating $T_{a}$.

Definition IV.2 (Global Errors). With respect to Clark's formulation, edge $e_{i}$ in a cutset $\Sigma$ is said to have global errors, iff its computed criticality $T_{i}$ differs from its true criticality and the edge does not exhibit local errors, i.e.,

$$
T_{i} \leq \tau_{i j} \quad\left\{\forall e_{j} \in \Sigma, \quad e_{j} \neq e_{i}\right\}
$$

and $T_{i}$ is in error

By definition, global errors can either overestimate or underestimate the criticality. For the toy example, $T_{a}$ is underestimated by 0.567 . Note that the value of $T_{a}$ is consistent with Eq. 4, since both $\tau_{a b}=1.0$ and $\tau_{a c}=0.921$ are greater than $T_{a}=0.356$

In summary, although local and global errors result from the linear approximation of Clark's $M A X_{\theta}$ operation, local errors are an artifact of the manner in which we compute global criticalities of edges in a cutset whereas global errors are more fundamental, overestimating or underestimating the true criticality of an edge in a cutset.

\section{Clustering Based Pruning and Ordering}

Definition V.1 (Dominant and Non-dominant Edges). An edge $e_{i}$ in set $\Sigma$ is said to be dominant iff its local criticality with respect to all other edges in $\Sigma$ is above a threshold $\varepsilon$, i.e.

$$
\tau_{i j}>\varepsilon \quad\left\{\forall e_{j} \in \Sigma, \quad e_{j} \neq e_{i}\right\}
$$

Otherwise, $e_{i}$ is said to be non-dominant in $\Sigma$, i.e.,

$$
\left\{\exists e_{j} \in \Sigma, \quad e_{j} \neq e_{i}\right\} \quad: \quad \tau_{i j} \leq \varepsilon
$$

Definition V.2 (Mutually-dominant Edges). A set of edges $\Sigma$ are said to be mutually dominant iff each edge in $\Sigma$ is dominant, i.e.,

$$
\tau_{i j}>\varepsilon \quad\left\{\forall e_{i}, e_{j} \in \Sigma, \quad e_{j} \neq e_{i}\right\}
$$

As seen in the previous section, non-dominant edges (like $b$ in Fig. 2) in a cutset exhibit local errors. Moreover, they also contribute to global errors of other edges in the cutset (like $a$ in Fig. 2). To avoid the bulk of these errors we propose to prune the cutset, eliminating its non-dominant edges from competing and injecting errors in global criticality computations.

Pruning is justified by Eq. 4, wherein eliminating edge $e_{i}$ with local criticality lower than a sufficiently small threshold value $\varepsilon$ does not hurt global criticality computations because $T_{i} \leq \varepsilon$. The benefits are accentuated in cutsets with dominant edges with large global criticalities, since the sum of global criticalities across a cutset equals 1.0 (implying that many edges have very small local criticalities).

It must be pointed out, that not every edge with global criticality below $\varepsilon$ can be eliminated by pruning because its local criticality might not be smaller than $\varepsilon$. Such edges cause global errors using the linear $M A X_{\theta}$ operation.

\section{A. $\mathbf{n C 2}$ Cutset Pruning}

A straightforward approach to pruning a cutset would be to perform pairwise comparisons of edges, eliminating those that have a minimal local criticality less than a predefined threshold $\varepsilon$. The main drawback of this approach is its quadratic time complexity of $O\left(n^{2}\right)$, due to $n C 2$ local criticality computations, where $n$ is the number of edges in the cutset. This can be prohibitive for large circuits.

\section{B. Clustering Based Cutset Pruning and Ordering}

To overcome the quadratic runtime complexity overhead of the $\mathrm{nC} 2$ approach we present a new clustering-based pruning heuristic based on the $K$-center clustering algorithm of [4]. Algorithm 3 describes the procedure. The basic idea is to prune a cutset into a set of mutually dominant edges by removing its non-dominant edges. Variables used in the algorithm are,
$d_{i \kappa}$ : Distance of an object $i$ from its cluster center $\kappa$ is its local criticality $\tau_{i \kappa}$
$r_{\sigma}$ : Radius of cluster $\sigma$ is distance of the object farthest from $\kappa$, i.e., $r_{\sigma}=$
$M A X\left(d_{i \kappa}\right) \forall i \in \sigma$
$R_{\sigma}$ : Border $R$ of cluster $\sigma$ is object with the maximum distance from $\kappa$, i.e.,
$R_{\sigma}=j: d_{j \kappa}=r_{\sigma}$

We first prune cutset $\Sigma$ with respect to seed $\chi$, chosen as the object with maximum mean in $\Sigma$. Steps 5-8 of Algorithm 3 compute new clusters from existing ones (Algorithm 4) until no cluster has size exceeding $S$. Finally, cutset $\Sigma$ is replaced by the remaining un-pruned objects.

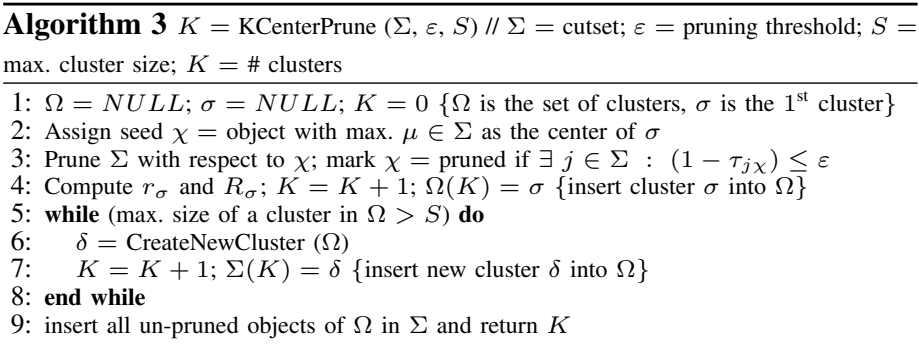

Intuitively, $\chi$ in Algorithm 4 is the object upon which its center has the lowest degree of domination (Def II.6) and hence a good candidate to facilitate pruning of other edges in the cutset. Step 3 uses $\chi$ to prune objects $j$ (with local criticality with respect to $\chi$ less than $\varepsilon$ ) from their respective clusters. Alternatively, if $\chi$ has a higher degree of domination over $j$ compared to its current center $\kappa, j$ is removed from its cluster and inserted into new cluster $\delta$ (Step 3). Intuitively, a greater degree of domination between two edges results in smaller global errors in $M A X_{\theta}$. We return $\delta$ after adjusting it radius and border and those of all currently existing clusters in $\Omega$ (Step 4). The algorithm has the following properties.

\begin{tabular}{l} 
Algorithm $4 \delta=$ CreateNewCluster $(\Omega) / / \Omega=$ set of clusters; $\delta=$ new cluster \\
\hline 1: Assign $\chi=$ border of cluster with max. radius as center $\kappa$ of new cluster $\delta$ \\
2: Prune $\Omega$ with respect to $\chi$; mark $\chi=$ pruned if $\exists j \in \Omega:\left(1-\tau_{j \chi}\right) \leq \varepsilon$ \\
3: if $\exists j \in \Omega: \tau_{j \chi}<d_{j \kappa}$, remove $j$ from its current cluster and insert into $\delta$ \\
4: compute radius and border for $\delta$ and all existing clusters in $\Omega$; return $\delta$
\end{tabular}

Property V.1. At any iteration, all objects in $\Omega$ (excluding cluster centers marked pruned) are dominant with respect to all existing cluster centers.

Proof: To avoid pruning in Line 3 of Algorithm 3, objects must be dominant with respect to the seed ( $1^{\text {st }}$ cluster center). Moreover, every object $j$ is compared with every newly added cluster center (over the entire run of the algorithm, all cluster centers) in Line 2 of Algorithm 4. Clearly, $j$ must be dominant with respect to these centers to avoid being pruned. Moreover, Line 3 of Algorithm 3 and Line 2 of Algorithm 4 compare every cluster center with every object for dominance. Although not removed from $\Omega$, centers are marked pruned if they are non-dominant with respect to other cluster objects.

Property V.2. With $S=1, \operatorname{KCenterPrune}(\Sigma, \varepsilon, 1)$ returns a set of mutually dominant edges (see Def.V.2) in $\Sigma$.

Proof: With $S=1$ each cluster in $\Omega$ contains only one object, its cluster center. From Property V.1 above we know that that these are either marked pruned or are dominant with respect to other cluster centers. It follows from step 9 of Algorithm 3 that $\Sigma$ contains mutually dominant objects.

Property V.3. For any cluster $\sigma \in \Omega$, its center $\chi$ has a higher degree of domination over its members than any other cluster center $\kappa$, i.e.,

$$
\tau_{\chi j}>\tau_{\kappa j} \quad\{\forall j \in \sigma, \kappa \in \Sigma, \quad \kappa \neq \chi\}
$$


TABLE II

CRITICALITY RUN-TIMES AND ERRORS FOR VARIOUS BENCHMARKS; $\varepsilon=5 \%$ AND $N_{l s}=1000$

\begin{tabular}{|c|c|c|c|c|c|c|c|c|c|c|}
\hline \multirow[t]{2}{*}{ Metric } & \multirow{2}{*}{$\begin{array}{l}\text { Pruning } \\
\text { Scheme }\end{array}$} & \multicolumn{9}{|c|}{ Benchmark } \\
\hline & & s27 & s1196 & s5378 & s9234 & s13207 & $\mathrm{s} 15850$ & s38417 & s35932 & s38584 \\
\hline \multirow{4}{*}{$\begin{array}{l}\max . \\
\delta \%\end{array}$} & ZSC & 3.84 & 2.25 & 47.99 & 45.79 & 38.11 & 58.49 & 40.82 & 24.99 & 43.56 \\
\hline & $\mathrm{nC2}$ & 3.84 & 2.99 & 3.13 & 21.97 & 2.65 & 6.43 & 37.82 & 16.68 & 18.65 \\
\hline & CPSC & 3.84 & 2.99 & 3.13 & 23.65 & 5.30 & 5.49 & 37.82 & 16.12 & 18.65 \\
\hline & CPSC+TGR+LS & 3.84 & 2.99 & 3.13 & 2.92 & 5.21 & 2.37 & 2.17 & 2.79 & 15.82 \\
\hline \multirow{4}{*}{$\begin{array}{l}\text { run } \\
\text { time } \\
\text { (sec) }\end{array}$} & $\mathrm{ZSC}$ & 0.00 & 0.03 & 0.16 & 0.30 & 0.45 & 0.54 & 2.51 & 2.71 & 3.06 \\
\hline & $\mathrm{nC} 2$ & 0.00 & 0.02 & 0.39 & 0.71 & 2.31 & 2.19 & 43.53 & 55.39 & 51.76 \\
\hline & CPSC & 0.00 & 0.01 & 0.02 & 0.05 & 0.07 & 0.09 & 0.27 & 0.34 & 0.25 \\
\hline & CPSC+TGR+LS & 0.00 & 0.00 & 0.03 & 0.08 & 0.09 & 0.11 & 0.34 & 0.37 & 0.34 \\
\hline \multirow{4}{*}{$\eta$} & ZSC & 7 & 202 & 593 & 644 & 1329 & 1688 & 2821 & 7340 & 5680 \\
\hline & $\mathrm{nC} 2$ & 2 & 1 & 2 & 11 & 4 & 4 & 5 & 49 & 11 \\
\hline & CPSC & 2 & 1 & 2 & 11 & 4 & 4 & 5 & 49 & 11 \\
\hline & CPSC+TGR+LS & 2 & 1 & 2 & 11 & 4 & 4 & 5 & 49 & 11 \\
\hline
\end{tabular}

Proof: This is evident from Step 3 of Algorithm 4. Each object in $\Omega$ is compared with the new cluster center $\chi$. The condition $\tau_{j \chi}<d_{j \kappa}$ is equivalent to $\tau_{\chi j}>\tau_{\kappa j}$, i.e., the new cluster center $\chi$ has a higher degree of domination over object $j$ than its current cluster center $\kappa$.

Property V.4. For a cutset $\Sigma$ of size $n$ and $K$ clusters returned, KCenterPrune takes $O(n K)$ time.

Proof: A single run of Algorithm 4 compares every object in $\Sigma$ with center $\chi$ of the new cluster $\delta$, taking $O(n)$ time. Since each iteration in Algorithm 3 returns a new cluster, with $K$ clusters returned, the overall runtime is $O(n K)$.

\section{CPSC: Clustering Based SC Algorithm}

Using Algorithm 3 for SC computation is straightforward. Step 2 in Algorithm 5 derives a set of mutually dominant edges from cutset $\Sigma$, facilitated using Property V.2 and orders $\Sigma$, facilitated by Property V.3. Such an ordering cannot be obtained with the $\mathrm{nC} 2$ pruning strategy of Section V-A. Property V.4 ensures that with a small number of dominant edges $K$ in a cutset, the algorithm runs in linear time.

\section{Localized Sampling and Timing Graph Reduction}

To tackle edges that lead to global errors (Def. IV.2), we perform a quick localized Monte Carlo sampling of the edges in cutset $\Sigma$ after pruning. We generate $N_{l s}$ samples of the $k$ independent identically distributed Gaussians, $k$ being the number of PCs (Def. II.4). Each sample is used in instantiating $e_{i}\left(\forall e_{i} \in \Sigma\right)$, from which we compute the edge in $\Sigma$ with the maximum delay. By keeping count of the number of samples for which an edge in $\Sigma$ has maximum delay, we can compute its global criticality probability.

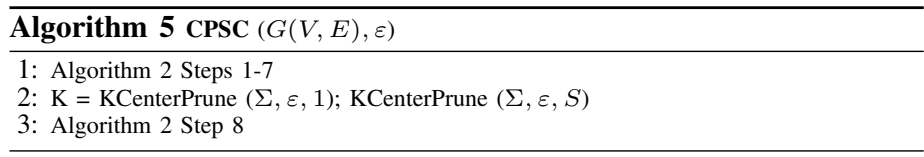

The runtime of LS increases with the number of samples $N_{l s}$ and the depth $L$ of the timing graph $G$. Reducing $N_{l s}$ reduces the accuracy of criticality computation but $L$ has no effect on the accuracy. Moreover, the criticality of a node in $G$ is equivalent to the sum of its fanin edge or fanout edge criticalities. Exploiting this property, we perform a timing graph reduction (TGR) procedure on nodes with a single fanin or fanout. A straightforward and practical example of this reduction is an inverter chain

Table III shows the effect of TGR on the depth (number of levels) $L$ and maximum cutset size $\eta$ on the three largest benchmark circuits. Column 2 shows the size of the circuit. As their names imply, columns w/ red. and w/o red. are results with and without TGR respectively, applied to $G$.

TABLE III

EFFECT OF TGR ON CIRCUIT DEPTH $L$ AND MAXIMUM CUTSET SIZE $\eta$

\begin{tabular}{|l|l|l|l|l|c|}
\hline Benchmark & \# of & \multicolumn{2}{|c|}{$L$} & \multicolumn{2}{|c|}{$\eta$} \\
\cline { 3 - 6 } & Gates & w/o red. & w/ red & w/o red. & w/ red \\
\hline s38417 & 22179 & 51 & 13 & 2821 & 6638 \\
\hline s35932 & 16065 & 33 & 10 & 5473 & 10742 \\
\hline s38584 & 19253 & 60 & 19 & 5680 & 10374 \\
\hline
\end{tabular}

\section{RESULTS}

Our algorithms were implemented in C++ on top of an SSTA engine and exercised on the ISCAS89 benchmarks, with parameter values corresponding to the 100nm technology node [7]. Experiments were conducted on a Linux PC with a 3.0-GHz CPU and 2GB RAM. We compared four schemes with Monte Carlo simulations using 10000 samples, shown in Table II.
The first scheme is the ZSC approach in Algorithm 2. Scheme nC2 implements the pairwise pruning strategy of Section V-A with a pruning threshold $\varepsilon$ of $5 \%$. CPSC implements Algorithm 5 using our clustered pruning and ordering technique. Finally, CPSC+LS+TGR performs clustered pruning on the reduced timing graph (TGR) and computes criticalities using the LS procedure with $N_{l s}=1000$ samples. Row max. $\delta$ reports the maximum difference between the edge criticality computed using any of the above mentioned schemes and the Monte Carlo simulations, run-time reports the running time in seconds and $\eta$ reports the maximum number of edges in any cutset of the timing graph after pruning. We exclude the times for SSTA and generating samples in the LS scheme.

From Table II, ZSC which computes criticalities using Clark's $M A X_{\theta}$ formulation results in large errors (the largest being 58\%). Since ZSC fundamentally does not differ from [10], we expect to see errors of similar magnitude. CPSC with cutset pruning and ordering does better than ZSC in accuracy and runtime. For circuits exhibiting large global errors, ordering is insufficient to reduce these errors. Rows in bold compare ZSC with CPSC+TGR+LCS. The combined approach greatly reduces errors with negligible increase in runtime compared to CPSC. For the 3 large benchmarks we obtain about an order of magnitude difference run-times of ZSC and the combined approach. Most circuits have errors below 5\%, except for s38584. On investigation, it was found that for large fanout structures, path delays themselves (computed in terms of the PCs) contained large errors and hence the LS procedure does not completely eliminate global errors. In terms of the efficacy of our pruning strategy, as expected we vastly outperform the $\mathrm{nC} 2$ procedure in run-time (about two orders of magnitude for the larger benchmarks). Moreover, each circuit contained an identical number of edges remaining in the cutsets using the $\mathrm{nC} 2$ and CPSC pruning strategies.

\section{REFERENCES}

[1] H. Chang and S. S. Sapatnekar, "Statistical timing analysis considering spatial correlations using a single PERT-like traversal," in Proceedings of the 2003 IEEE/ACM International Conference on Computer-Aided Design. IEEE Computer Society, 2003, p. 621.

[2] K. Chopra, S. Shah, A. Srivastava, D. Blaauw, and D. Sylvester, "Parametric yield maximization using gate sizing based on efficient statistical power and delay gradient computation," in Proceedings of the 2005 IEEE/ACM International Conference on Computer-Aided Design. IEEE Computer Society, 2005, pp. 1023-1028.

[3] C. E. Clark, "The greatest of a finite set of random variables," Operations Research, vol. 9, no. 2, pp. 145-162, Mar-Apr 1961.

[4] T. F. Gonzalez, "Clustering to minimize the maximum intercluster distance," Theoretical Computer Science, vol. 38, no. 2-3, pp. 293-306, 1985.

[5] X. Li, J. Le, M. Celik, and L. T. Pileggi, "Defining statistical sensitivity for timing optimization of logic circuits with large-scale process and environmental variations," in Proceedings of the 2005 IEEE/ACM International Conference on Computer-Aided Design. IEEE Computer Society, 2005 pp. $844-851$.

[6] S. R. Nassif, "Design for variability in DSM technologies," in Proceedings of the 1st International Symposium on Quality of Electronic Design. IEEE Computer Society, 2000, p. 451.

[7] Predictive technology model (PTM). [Online]. Available: http://www.eas. asu.edu/ $\sim \mathrm{ptm} /$

[8] D. Sinha, H. Zhou, and N. V. Shenoy, "Advances in computation of the maximum of a set of random variables," in Proceedings of the 7th International Symposium on Quality Electronic Design, Mar. 27-29, 2006.

[9] C. Visweswariah, K. Ravindran, K. Kalafala, S. G. Walker, and S. Narayan, "First-order incremental block-based statistical timing analysis," in Proceedings of the 41st Annual Design Automation Conference. ACM Press, 2004, pp. $331-336$.

[10] J. Xiong, V. Zolotov, N. Venkateswaran, and C. Visweswariah, "Criticality computation in parameterized statistical timing," in Proceedings of the 43rd Design Automation Conference. ACM Press, 2006, pp. 63-68.

[11] T. Yoshimura and E. S. Kuh, "Efficient algorithms for channel routing," IEEE Transactions on Computer-Aided Design of Integrated Circuits and Systems, vol. 1, no. 1, pp. 25-35, Jan. 1982. 